Journal of

Synchrotron

Radiation

ISSN 0909-0495

Received 22 August 2004

Accepted 23 November 2004

\section{An internet-based synchrotron experiment for students measuring the $X$-ray magnetic circular dichroism of a PtFe alloy}

\author{
Michael Paulus, ${ }^{\text {a* }}$ Robert Fendt, ${ }^{\text {a }}$ Christian Sternemann, ${ }^{a}$ Christian Gutt, ${ }^{\text {a }}$ \\ Heinz Hövel, ${ }^{a}$ Martin Volmer, ${ }^{a}$ Metin Tolan ${ }^{a}$ and Klaus Wille ${ }^{b}$ \\ anstitute of Physics, University of Dortmund, D-44221 Dortmund, Germany, and ${ }^{\mathbf{b}}$ DELTA, \\ University of Dortmund, D-44221 Dortmund, Germany. E-mail: michael.paulus@uni-dortmund.de
}

(C) 2005 International Union of Crystallography Printed in Great Britain - all rights reserved

\begin{abstract}
A new internet-based synchrotron experiment for students is presented. A polarimeter and computer software have been developed for measuring via the internet the X-ray magnetic circular dichroism of PtFe around its $\mathrm{Pt} L_{\mathrm{II}}$ and $L_{\mathrm{III}}$ absorption edges. From the experiment, students can examine the X-ray magnetic circular dichroism of a thin PtFe foil utilizing circular-polarized synchrotron radiation emitted by the superconducting asymmetric wiggler at the synchrotron radiation source DELTA of the University of Dortmund.
\end{abstract}

Keywords: XMCD; monochromators; beamlines; internet.

\section{Introduction}

Today, X-rays generated in third-generation synchrotron sources provide one of the most powerful tools for studying many aspects of condensed matter physics. Most of the research groups using synchrotron radiation have a strong university background, i.e. they do not only contribute to the research but also educate students in their specialized areas of research. At least in their first years at university, students learn about the basic principles of the generation of X-rays and their characteristic spectral properties. As X-ray tubes are relatively easy to handle, there are many student experiments in practical courses, e.g. the measurement of Debye-Scherrer rings. In contrast, the properties of synchrotron radiation are treated from a more theoretical point of view in the lectures, while practical applications or the possibility of performing synchrotron experiments on their own is virtually excluded for the vast majority of students. Therefore, a new type of internet-based synchrotron experiment for students has been developed, and is presented here.

The experiment is located at the beamline SAW2 (BL9) of the Dortmund Electron Accelerator (DELTA). The internet experiment has been developed within the framework of the Physik 2000 project. This project, initiated by the German Federal Ministry of Education and Research, is aiming towards implementing new media techniques in the traditional physics courses of German universities. The experiment should cover typical properties of synchrotron radiation such as the high brilliance, the tunable photon energy and the availability of circular polarization. In addition, an experimental technique should be used which is less common in traditional physics courses but shows how successful synchrotron radiation can be used in solid state physics. The experiment focuses on measuring the X-ray magnetic circular dichroism (XMCD) which is a powerful element-specific technique for investigating the magnetic moment of an element with respect to its spin and orbital momentum and its relative orientation. This internet experiment will be based on an early study of the $L_{\mathrm{II}}$ and $L_{\mathrm{III}}$ absorption edges of $\mathrm{Pt}$ in a PtFe alloy (Schütz et al., 1989; Ebert et al., 1989). Owing to the fact that the PtFe system shows an extremely large XMCD signal, it turned out to be an ideal sample for the students' experiment. This paper gives an example of how the internet could be used as an educational tool giving the students the opportunity to perform an experiment at a synchrotron radiation source. It is out of the scope of the experiment to give a state-of-the-art account of how X-ray magnetic circular dichroism is measured.

The paper is organized as follows. In $\S 2$ we briefly present the physics of XMCD. In $\$ 3$ the experimental set-up is presented while $\$ 4$ contains a detailed description of the internet-based control system of the experiment. Typical experimental results and a conclusion are given in $\S 5$.

\section{X-ray magnetic circular dichroism}

Several techniques exist for measuring the magnetic properties of materials. Most of them are sensitive to the total magnetization of the measured system and cannot discern between the contributions of different atoms in an alloy or multilayer. Moreover, the small quantity of material present in many technologically interesting samples, such as magnetic nanostructures, necessitates a very sensitive measuring method. One of the most powerful techniques combining these different properties is XMCD. The aim of XMCD experiments is to measure the difference of the absorption of 
right- and left-handed circularly polarized photons in a magnetized target as a function of the photon energy. The quantities measured in such experiments are the spin-dependent absorption coefficients $\mu^{+}$and $\mu^{-}$, where $\mu^{+}$denotes the absorption coefficient of photons with spin parallel and $\mu^{-}$ with spin antiparallel to the sample magnetization. Within an XMCD experiment $\mu^{ \pm}$can be determined by turning either the direction of the magnetic field or changing the polarization of the radiation and measuring the transmitted photon intensity normalized to the incident photon intensity using, for example, ionization chambers.

XMCD was predicted by Erskine \& Stern (1975), reporting that X-ray absorption experiments performed with circularly polarized light can supply magnetic information about the initial state of the absorption process. Since then, XMCD has attracted great interest as a useful tool for investigating magnetic states (Schütz et al., 1987, 1993; Schütz \& Wienke, 1989; Rüegg et al., 1991; Vogel et al., 1997; Poulopoulos et al., 2002; Ederer et al., 2002; Takahashi \& Igarashi, 2003; Wende et $a l ., 2003)$. Despite the fact that the technique is relatively new, it has already entered textbooks of X-ray physics (Lovesey \& Collins, 1996; Als-Nielsen \& McMorrow, 2001; Beaurepaire et al., 2001). Since XMCD can be observed at absorption edges, the experiments become highly element specific. As the $\mathrm{XMCD}$ effect is very sensitive, it allows for the determination of a very small quantity of materials. This sensitivity enables, for example, the study of nano-structured materials present in modern magnetic storage devices.

An especially large XMCD effect is observed at the $L_{\mathrm{II}}$ and $L_{\text {III }}$ edges in the ferromagnetic alloy PtFe (Schütz et al., 1989; Ebert et al., 1989). At these edges, values of $A_{L_{\mathrm{II}}} \simeq 22 \%$ and $A_{L_{\mathrm{III}}} \simeq 13 \%$ have been reported, where $A$ denotes the relative difference of spin-dependent absorption via

$$
A=\Delta \mu /\left[\mu^{+}+\mu^{-}\right]
$$

Hereby, $\Delta \mu=\mu^{+}-\mu^{-}$describes the dichroic and $\mu^{+}+\mu^{-}$ the isotropic signal. First theoretical descriptions of the XMCD by Schütz et al. (1989) used a two-step model where the absorption process is split into two parts, the production of a spin-polarized photoelectron by absorbing circular-polarized photons and the accommodation of the photoelectron into an unoccupied spin-polarized state. In this case, $A$ can be described theoretically by

$$
A=\left\langle\sigma_{z}\right\rangle \Delta \rho_{\mathrm{s}} / \rho .
$$

Here $\left\langle\sigma_{z}\right\rangle$ denotes the energy-independent spin polarization of the photoelectron, $\Delta \rho_{\mathrm{s}}$ the spin polarization and $\rho$ the total density of the populated empty states.

Assuming free atoms one obtains $\left\langle\sigma_{z}\right\rangle_{p_{1 / 2}}=-0.5$ for the initial $p_{1 / 2}$ and $\left\langle\sigma_{z}\right\rangle_{p_{3 / 2}}=0.25$ for the initial $p_{3 / 2}$ core state. In this case, transitions $2 p_{1 / 2} \rightarrow$ $5 d_{3 / 2}$ and $2 p_{3 / 2} \rightarrow 5 d_{5 / 2}$ are considered only and a ratio between $A_{L_{\text {II }}}$ and $A_{L_{\text {III }}}$ of -2 is obtained. Within the limits of

Figure 1 text for details). this model the measured XMCD data of the Pt $L$ edges leads to a determination of the spin polarization of the unoccupied $5 d$ bands. Ebert \& Zeller (1990) have discussed the two-step model utilizing relativistic calculations which include spinorbit coupling of the initial and final states. They pointed out that in the case of the PtFe system the assumption of a constant photoelectron polarization, the limitation to $2 p_{1 / 2} \rightarrow 5 d_{3 / 2}$ and $2 p_{3 / 2} \rightarrow 5 d_{5 / 2}$ transitions and a simplified treatment of the unoccupied spin-polarized states lead to a proper description of the experimental data. However, in general, sum rules have to be used in analyzing XMCD from $L$ edges in order to determine orbital and spin moments along with their relative orientation [for a detailed description see, for example, Wende et al. (2003) and Ebert \& Zeller (1990)].

The application of the sum rules requires the determination of both $\Delta \mu_{L_{\mathrm{II}}}$ and $\Delta \mu_{L_{\mathrm{III}}}$. Because of the restricted time to perform the internet experiment, the students have to focus on either the $\mathrm{Pt}_{L_{\text {II }}}$ or $\mathrm{Pt}_{L_{\text {III }}}$ edge. Therefore, the student's analysis of the experimental results will be focused on the determination of the relative difference of spin-dependent absorption $A$ and its interpretation will be carried out on the basis of the simple two-step model by Schütz et al. (1989).

\section{Experimental set-up}

\subsection{Synchrotron source and beamline}

DELTA is a synchrotron radiation source located at the University of Dortmund, Germany. The synchrotron runs at an energy of $1.5 \mathrm{GeV}$ with a current of $120 \mathrm{~mA}$ and typical lifetimes of $10 \mathrm{~h}$ (Tolan et al., 2003). The synchrotron radiation is produced by dipole magnets, two undulators and by a superconducting asymmetric wiggler (SAW). The SAW is used for the internet experiment. The asymmetric arrangement of the magnetic units in the wiggler produces a high-intensity circularly polarized X-ray beam. The polarization can be changed from left-handed to right-handed by changing the view on the orbit (i.e. using radiation below or above the electron orbit). Three beamlines are located at the SAW. The internet experiment is built up at beamline SAW2 (BL9). A schematic drawing of the beamline is displayed in Fig. 1. The optical devices consist of two gold-coated mirrors and a $\mathrm{Si}(311)$ double-crystal monochromator. The beam is vertically focused by the sagittally bent second monochromator crystal

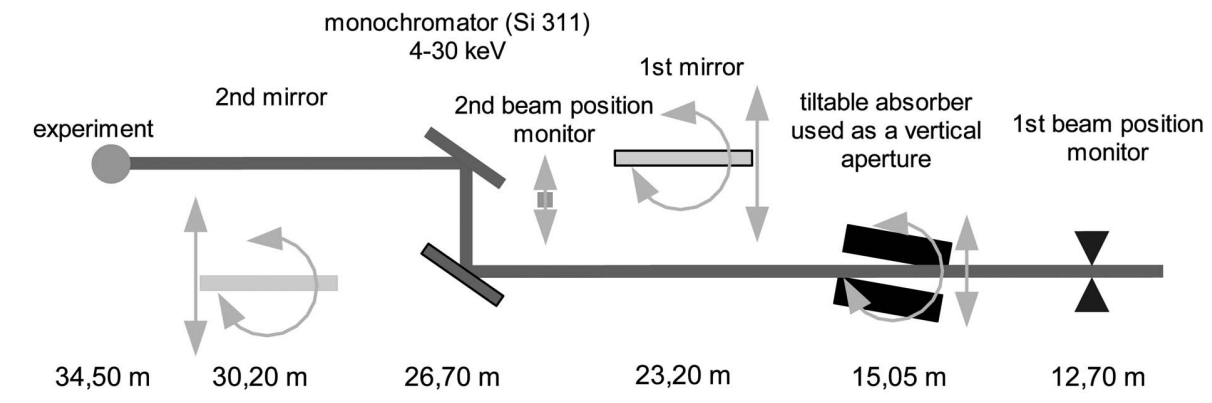

Schematic drawing of the beamline SAW2 (BL9) at the synchrotron radiation source DELTA (see 
whereas the horizontal focusing is performed using the second gold mirror. The monochromator provides an energy resolution of $\Delta E / E \simeq 10^{-4}$. The energy range of the radiation is between 4 and $30 \mathrm{keV}$. Two experimental end-stations are available: a spectrometer in Rowland geometry used for resonant and non-resonant inelastic X-ray scattering experiments, and a six-circle diffractometer utilized for powder and surface diffraction. The XMCD set-up has been built up as an extension of the Rowland spectrometer and will be described in detail later. The gold-coated mirrors have not been used during the experiment. Furthermore, the polarization of the radiation will be partially reduced owing to the Bragg reflection of the monochromator crystals which has to be accounted for. This reduction depends on the Bragg angle $\theta$ and is approximated by

$$
P^{\prime}=\left[\frac{2 \cos (2 \theta)}{1+\cos (2 \theta)}\right]^{2} P=R_{\mathrm{mono}} P,
$$

where $P^{\prime}$ and $P$ denote the degree of polarization behind and before the monochromator, respectively (Zachariasen, 1967). This implies that the degree of polarization for photons of energy below $8 \mathrm{keV}$ is reduced by more than $50 \%$ by the monochromator. Thus the $L_{\mathrm{II}}$ and $L_{\mathrm{III}}$ absorption edges of $\mathrm{Pt}$ are ideal candidates for the experiment owing to their rather high excitation energies leading to a minor reduction of the degree of circular polarization of $17 \%$ and $23 \%$, respectively.

\subsection{Sample and polarimeter}

Absorption experiments require rather thin samples. Here a thin foil of a rolled ferromagnetic PtFe alloy (impurity concentration of $3 \% \mathrm{Pt}$ ) was used. The Pt $L_{\mathrm{II}}$ and $L_{\mathrm{III}}$ edges are at energies of 11564 and $13273 \mathrm{eV}$. Besides the favourable strong XMCD effect and the easily accessible energies of the Pt absorption edges the use of a ferromagnetic PtFe alloy has the additional advantage of a rather high Curie temperature of $T_{\mathrm{C}} \simeq 1100 \mathrm{~K}$, which allows measurements at room temperature.

In order to achieve a large XMCD effect the direction of the magnetization should be parallel or antiparallel to the wavevector of the incident photons. However, owing to the use of a thin foil the magnetization of the PtFe sample is always parallel to the surface of the foil (see Fig. 2a). An angle of $\alpha=30^{\circ}$ between the incident beam and the sample surface yields a good compromise between the XMCD effect and the absorption within the foil. In this geometry the XMCD effect is reduced by a factor of 0.87 only, while the sample thickness increases by a factor of two in comparison with $\alpha=90^{\circ}$. The absorption lengths of the PtFe foil at the $L_{\mathrm{II}}$ and $L_{\mathrm{III}}$ absorption edges are $10 \mu \mathrm{m}$ and $11 \mu \mathrm{m}$, respectively, so that a sample of about $10 \mu \mathrm{m}$ thickness is used. In the internet experiment the direction of circular polarization is fixed by choosing one angle of view on the electron orbit and the $\mathrm{XMCD}$ effect is measured by changing the direction of the external magnetic field.

The sample chamber is an aluminium cylinder of diameter $23 \mathrm{~cm}$ and height $10 \mathrm{~cm}$ with large Kapton windows. The whole sample chamber can be evacuated to pressures of $10^{-3}$ mbar, thus reducing the scattering of X-rays by air considerably. However, the vacuum prevents the use of an aircooled magnet inside the chamber. Therefore, the magnetic coils are located outside the sample cell allowing a cooling by air. The coils are wrapped around an iron yoke $(40 \mathrm{~mm} \times$ $40 \mathrm{~mm}$ ) which is led through the cell to the sample (see Fig. 2b). The sample holder is placed in a gap of length $20 \mathrm{~mm}$ in the iron yoke. Measurements utilizing a Hall probe showed that the magnitude of the magnetic field varies by less than $1 \%$ within the gap. Thus, a homogeneous field is obtained. Because of the angle $\alpha=30^{\circ}$ between the direction of the magnetic field and the X-ray beam, small windows of diameter $6 \mathrm{~mm}$ had to be drilled into the iron yoke. The magnetic coil consists of 800 windings producing a magnetic field of $150 \mathrm{mT}$ at a current of $2.5 \mathrm{~A}$. This magnetic field does not support a total magnetization of the sample which leads to an additional reduction of the XMCD effect. The power source is a bipolar power supply which can be steered by a control voltage provided by a D/A converter. To avoid any influence of the magnetic field on the ionization chambers an iron shielding is mounted around the polarimeter.

\section{Internet-based control of the experiment}

Running a synchrotron experiment via the internet demands a high level of computer security. The basic experimental control is performed using the software package SPECTRA (for details see http://www-hasylab.desy.de), which controls the monochromator, the detectors and the magnetic field of the polarimeter via VME crates. This beamline control software has been expanded to allow access to the SPECTRA
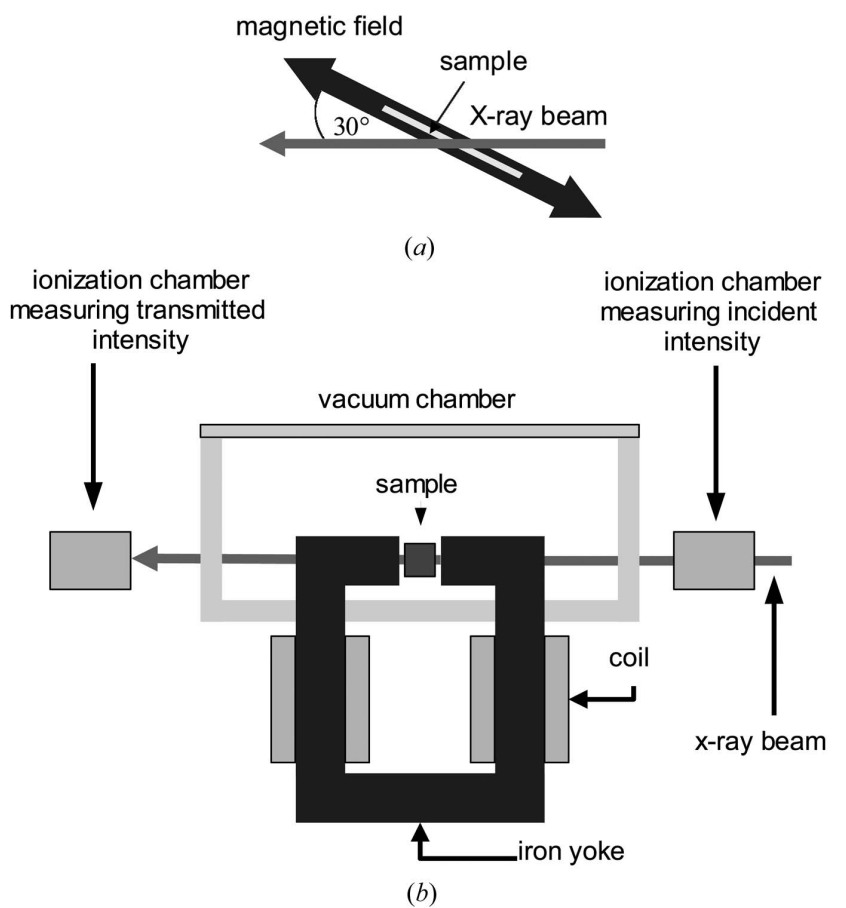

Figure 2

(b)

(a) Sample geometry. (b) Layout of the polarimeter. 
functions through the Perl programming language. A Perl driver programme has been written for this experiment. Both SPECTRA and the Perl driver run on the same PC with a Linux operating system. The Perl driver has access to a job database based on the SQL language. This database contains the user instructions and is installed on a separate computer with a UNIX operating system. The database entries are generated from a management server which is the only computer with direct connection to the internet. This management computer provides the web interface for the students based on java server pages.

An entry generated in the web interface by a student is processed according to the flow chart displayed in Fig. 3. The web server produces an entry in the SQL job database, which is checked every second by the Perl driver of the beamline control software. New entries are handled by the Perl driver and checked against the hardware limits. If the job entry is within these limits the Perl driver accesses the hardware components (e.g. to move the monochromator) directly via the SPECTRA system and returns the measured values.

By using the web interface the experiment is performed by the students in the following way:

(i) In a first step, the energy of the $L_{\mathrm{II}}$ or $L_{\mathrm{III}}$ absorption edge of $\mathrm{Pt}$ has to be found performing test scans. A reasonable

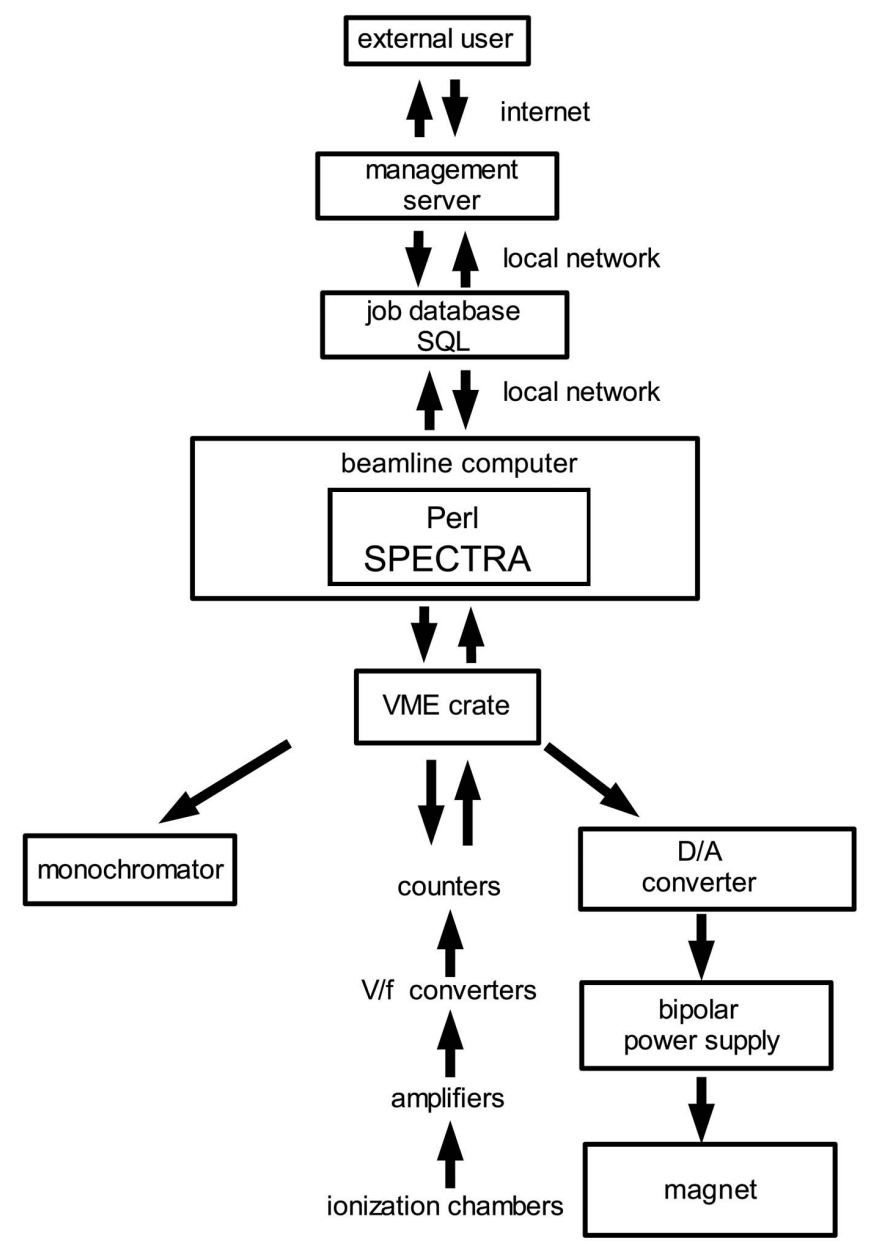

Figure 3

Flow chart of the internet-based control of the synchrotron experiment. scan range, step width and counting time have to be chosen by the students. During this first step the magnetic field is turned off.

(ii) In a second step, the absorption edge is measured with a sufficiently small energy step width and high counting statistics. Several single spectra have to be measured to check the single spectra for consistency.

(iii) After these experimental parameters are determined, the XMCD scan is started. During this scan the magnetic field is automatically switched by the Perl driver program after the counting time. The status of the storage ring is also shown in an extra window. An XMCD scan is measured four to six times in each experiment in order to monitor the quality of a single measurement.

(iv) A background scan is recorded starting at least a distance of $80 \mathrm{eV}$ away from the absorption edge. This background arises from low-energy absorption edges of the sample material.

Data analysis of the experiment is performed by the following steps:

(i) First, the data have to be normalized by dividing the transmitted intensity by the incident intensity monitored by the two ionization chambers before and behind the polarimeter.

(ii) To obtain the absorption coefficient the logarithm of the normalized data has to be taken.

(iii) The background has to be determined by linear regression from the background scan and subtracted from the XMCD scans.

(iv) The energy position of the absorption edge has to be estimated and compared with literature values.

(v) The XMCD effect has to be determined from the XMCD scans using equation (1). To obtain the XMCD signal scaled to $100 \%$ circularly polarized photons, the degree of circular polarization $P$ produced by the SAW and its reduction owing to the monochromator $R_{\text {mono }}$, the sample geometry $R_{\mathrm{g}}=$ 0.87 and the sample magnetization $R_{\mathrm{m}}=0.81$ have to be accounted for via $A_{\text {tot }}=A /\left(P R_{\text {mono }} R_{\mathrm{g}} R_{\mathrm{m}}\right)$.

\section{Results, conclusion and outlook}

The first students successfully performed the experiments in the summer of 2003; a second set of five experiments with external groups of two students each was performed in the summer of 2004. With repeated XMCD scans the duration of a typical experiment is about six hours. In the beginning of the experiment the students had difficulties in choosing correct estimates of the energy step widths and of the counting time. However, after help and advice by the local contact they were able to perform the experiment on their own. A direct and personal supervision was guaranteed by video conference. In addition, monitoring the students' computer screen by the local contact via virtual network computing (VNC) proved to be very helpful. Using VNC allowed a direct discussion of the spectra measured and a preliminary analysis of the XMCD data. Figs. 4(a) and 4(b) display the results of typical XMCD scans around the $L_{\mathrm{II}}$ and $L_{\mathrm{III}}$ absorption edges, respectively. 


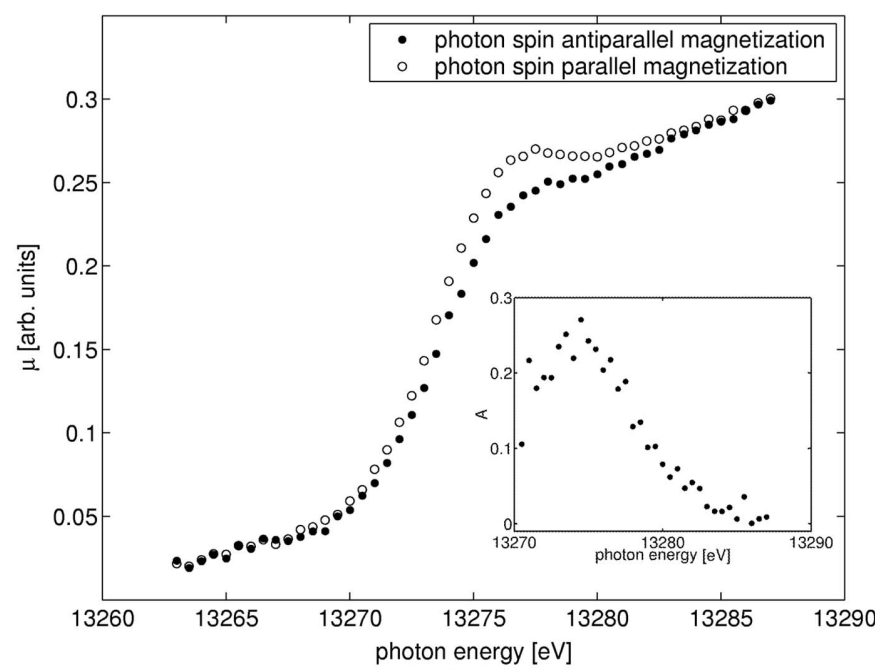

(a)

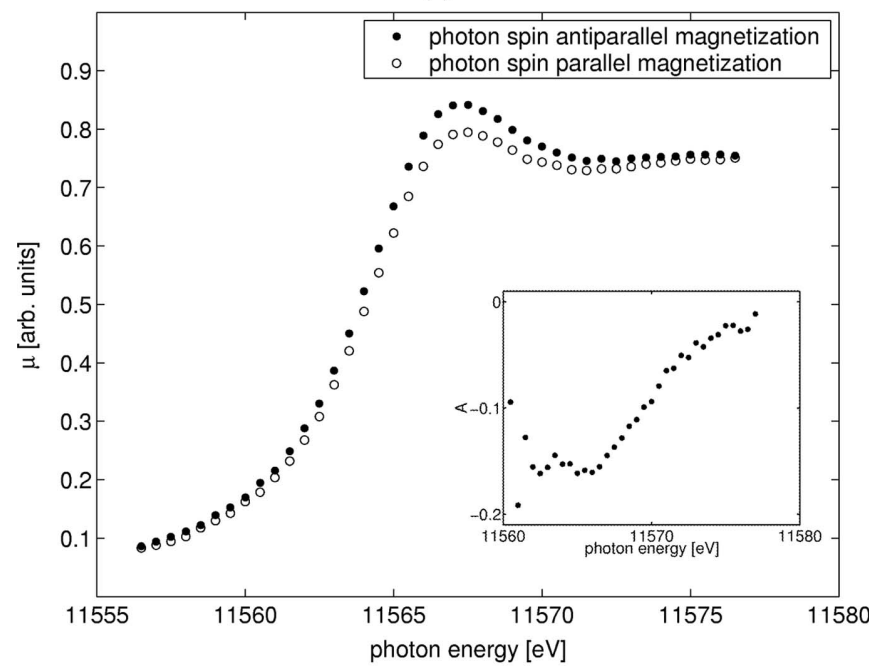

(b)

Figure 4

Results of typical $L$-edge scans for a PtFe sample: $(a)$ at the Pt $L_{\mathrm{II}}$ edge, (b) at the Pt $L_{\mathrm{III}}$ edge. The corresponding XMCD signal $A$ is presented in the insets.

The XMCD effect is clearly visible. The insets of Fig. 4 show the corresponding dichroic signals. The change in sign of the dichroic signal between the $L_{\mathrm{II}}$ and the $L_{\mathrm{III}}$ results is apparent, reflecting the change of spin polarization of the photoelectron. From their data the students calculated values of the relative difference of spin-dependent absorption $A$, which had to be corrected for the reduction factors discussed above to obtain the total XMCD effect $A_{\text {tot }}$. The final result has been compared with the original measurements of Schütz et al. (1989).

From the first internet experiments a very positive feedback has been obtained. The students pointed out that the combination of an internet experiment with a large-scale research facility was exciting and of interest for them. In summary, the internet-based synchrotron experiment allows students at universities to gain practical contact with the field of synchrotron-radiation-based research a long time before beginning their $\mathrm{PhD}$ studies. In the future, the experiment will be offered to all students of the universities participating in the Physik 2000 project. A better visualization of the experiment will be obtained after installing web cams showing the power supply, vacuum pressure and the voltmeters for the ionization chamber signals.

In conclusion, we have presented a new internet-based synchrotron experiment for students. A polarimeter and computer software have been developed for measuring, via the internet, the X-ray magnetic circular dichroism of a ferromagnetic PtFe alloy around its $\mathrm{Pt} L_{\mathrm{II}}$ and $L_{\mathrm{III}}$ absorption edges. By using circularly polarized synchrotron radiation emitted by the superconducting asymmetric wiggler at the synchrotron source DELTA, the students have been able to estimate the XMCD signal of the PtFe sample.

The authors thank P. Fischer (MPI Stuttgart) and J. Geißler (University of Würzburg) for providing the high-quality PtFe sample. This work is part of the university education program Physik 2000, sponsored by the BMBF. We gratefully acknowledge the help of our co-workers within this project and the enthusiasm of all students taking part in the experiments. The German Federal Ministry of Education and Research supported this work under contract No. 05 ET9 PEA.

\section{References}

Als-Nielsen, J. \& McMorrow, D. (2001). Elements of Modern X-ray Physics. New York: Wiley.

Beaurepaire, E., Schuerer, F., Krill, G. \& Kappler, J.-P. (2001). Magnetism and Synchrotron Radiation. Berlin: Springer.

Ebert, H., Drittler, B., Zeller, R. \& Schütz, G. (1989). Solid State Commun. 69, 485-487.

Ebert, H. \& Zeller, R. (1990). Phys. Rev. B, 42, 2744-2751.

Ederer, C., Komelj, M., Fähnle, M. \& Schütz, G. (2002). Phys. Rev. B, 66, 094413.

Erskine, J. L. \& Stern, E. A. (1975). Phys. Rev. B, 12, 5016.

Lovesey, S. W. \& Collins, S. P. (1996). X-ray Scattering and Absorption by Magnetic Materials. Oxford: Clarendon.

Poulopoulos, P., Scherz, A., Wilhelm, F., Wende, H. \& Baberschke, K. (2002). Phys. Status Solidi A, 189, 293-300.

Rüegg, S., Schütz, G., Fischer, P., Wienke, R., Zeper, W. B. \& Ebert, H. (1991). J. Appl. Phys. 69, 5655.

Schütz, G., Frahm, R., Wienke, R., Wilhelm, W., Wagner, W. \& Kienle, P. (1989). Rev. Sci. Instrum. 60, 1661-1665.

Schütz, G., Stähler, S., Knülle, M., Fischer, P., Parkin, S. \& Ebert, H. (1993). J. Appl. Phys. 73, 6430.

Schütz, G., Wagner, W., Wilhelm, W., Kienle, P., Zeller, R., Frahm, R. \& Materlik, G. (1987). Phys. Rev. Lett. 58, 737-740.

Schütz, G. \& Wienke, R. (1989). Hyperfine Interact. 50, 457.

Takahashi, M. \& Igarashi, J. (2003). Phys. Rev. B, 67, 245104.

Tolan, M., Weiss, T., Wille, K. \& Westphal, C. (2003). Synchrotron Rad. News, 16, 9-11.

Vogel, J., Fontaine, A., Cros, V., Petroff, F., Kappler, J.-P., Krill, G., Rogalev, A. \& Goulon, J. (1997). Phys. Rev. B, 55, 3663.

Wende, H., Scherz, A., Wilhelm, F. \& Baberschke, K. (2003). J. Phys. Condens. Matter, 15, 547-559.

Zachariasen, W. H. (1967). Theory of X-ray Diffraction in Crystals. New York: Dover. 